\title{
Combined Parametric and Worst-Case Circuit Analysis via Taylor Models
}

\author{
Riccardo Trinchero, Member, IEEE, Paolo Manfredi, Member, IEEE, Tongyu Ding, Student Member, IEEE, Igor \\ S. Stievano, Senior Member, IEEE
}

\begin{abstract}
This paper proposes a novel paradigm to generate a parameterized model of the response of linear circuits with the inclusion of worst-case bounds. The methodology leverages the so-called Taylor models and represents parameter-dependent responses in terms of a multivariate Taylor polynomial, in conjunction with an interval remainder accounting for the approximation error. The Taylor model representation is propagated from input parameters to circuit responses through a suitable re-definition of the basic operations, such as addition, multiplication or matrix inversion, that are involved in the circuit solution. Specifically, the remainder is propagated in a conservative way based on the theory of interval analysis. While the polynomial part provides an accurate, analytical and parametric representation of the response as a function of the selected design parameters, the complementary information on the remainder error yields a conservative, yet tight, estimation of the worst-case bounds. Specific and novel solutions are proposed to implement complexvalued matrix operations and to overcome well-known issues in the state-of-the-art Taylor model theory, like the determination of the upper and lower bound of the multivariate polynomial part. The proposed framework is applied to the frequencydomain analysis of linear circuits. An in-depth discussion of the fundamental theory is complemented by a selection of relevant examples aimed at illustrating the technique and demonstrating its feasibility and strength.
\end{abstract}

Index Terms-Circuit simulation, interval analysis, parameterized modeling, parametric simulation, Taylor models, tolerance analysis, uncertainty, worst-case analysis.

\section{INTRODUCTION}

The capability of accounting for the effects of stochastic and/or parametric variations of design parameters on circuit responses has become of paramount importance due to the increasing impact of variability in electronic components. Manufacturing process, temperature variations, uncontrollable parameters, and uncertain device characteristics, introduce possibly large variations of the circuit response that need to be accurately predicted during the design phase. Sensitivity/tolerance analysis, design exploration and design optimization therefore became crucial steps in the design workflow.

This work was partially supported by the Research Foundation Flanders (FWO-Vlaanderen).

R. Trinchero is with the Istituto Nazionale di Fisica Nucleare (INFN) Sezione di Torino, 10125 Torino, Italy (e-mail: riccardo.trinchero@to.infn.it).

P. Manfredi is with the Electromagnetics Group, Department of Information Technology, Ghent University, 9000 Gent, Belgium (e-mail: paolo.manfredi@ugent.be).

T. Ding and I. S. Stievano are with the EMC Group, Department of Electronics and Telecommunications, Politecnico di Torino, 10129 Torino, Italy (e-mail: tongyu.ding@polito.it, igor.stievano@polito.it).

Copyright (C) 2016 IEEE. Personal use of this material is permitted. However, permission to use this material for any other purposes must be obtained from the IEEE by sending an email to pubs-permissions@ieee.org.
Additionally, for critical (e.g., transport, industrial or medical) applications, it is often fundamental to determine the worstcase (WC) configuration that a possible uncertainty on the input parameters produces on the system output [1]-[5]. In order to set robust design margins, this has to be done in a conservative way, i.e., avoiding as much as possible underestimation.

In this framework, Monte Carlo (MC) is often used as a robust technique to explore the design space and assess the WC bounds, as well as to derive statistical information [6]-[8]. Yet, $\mathrm{MC}$ inherently provides underestimated WC responses, as only a subset of the possible realizations is actually considered. The determination of the "true" bounds could in fact be achieved only with an infinite number of samples. In practice, the number of simulations required to capture $\mathrm{WC}$ responses may be rather large as they often lie in the regions corresponding to distribution tails, and have therefore a low probability to occur. In addition to the aforementioned limitations, $\mathrm{MC}$ is "blind" and any relationship between the input parameters and the output quantities is unavoidably lost.

Parameterized macromodeling of linear networks was proposed to overcome the computational limitations of MC, while providing an explicit relationship between output responses and design variables [9]-[12]. The efficiency of the method stems from the limited number of simulation samples required to build the model, which is then re-used as an analytical and computationally cheap surrogate to perform, e.g., sensitivity analysis and/or optimization tasks. Statistical information is possibly obtained by MC sampling (e.g., [13]). However, the problem of underestimation of the WC bounds still exists, as the macromodel only provides a faster surrogate for the MCbased analysis.

More efficient techniques were proposed for stochastic macromodeling that rely on the theoretical framework of polynomial chaos (PC) and on the expansion of circuit responses in terms of orthogonal polynomials [14]-[19]. The advantage of PC lies in an optimal choice of the basis functions, which are more suitable to retrieve relevant stochastic information on the circuit response, such as statistical moments and probability density functions. This technique also yields, in fact, a parameterized model with respect to the uncertain variables. Nevertheless, $\mathrm{PC}$ provides probabilistic bounds only, which are not necessarily conservative, but rather given within a certain probability (e.g., three-sigma or 99\%-confidence bounds). Although WC bounds can be in principle extracted from the parametric PC representation [20], no information on the approximation error is available, and hence there is no 
guarantee that underestimation is avoided. It should be noted that PC is designed to provide better accuracy in the regions of the parameter space with higher probability density.

As opposed to the former class of probabilistic methods, inherently WC techniques were proposed to provide a conservative estimation of the upper and lower bounds of a system response. Specifically, interval methods are based on the representation of uncertain input parameters in terms of a bounded range of values the parameter can assume. The algebraic operations involved in the calculation of the system solution are then carried out in a modified manner, with the aim of providing a conservative bound of the actual result and eventually producing a guaranteed enclosure of the output responses. The most simple and basic tool is interval arithmetic or interval analysis (IA) [21], which is however a non-parametric tool. The lack of parameterization not only causes a loss of information on the input parameters, but it can also lead to large overestimation. Affine arithmetic (AA) is an enhanced representation with a corresponding, suitable modification of the pertinent algebraic operations [22]. It introduces an independent variable for each input parameter and keeps track of possible correlation between different quantities, thus considerably reducing overestimation [23]. Nonetheless, it can be regarded as a linear approximation with a modest accuracy. Applications of IA and AA to WC circuit simulation are found in [24]-[29].

Finally, Taylor models (TM) were proposed as a rigorous approach combining the strength of IA and of a higher-order parametric representation of the independent variables [30][32]. TMs represent variables in terms of multivariate Taylor polynomials, complemented by an interval remainder accounting for truncation errors. In the chain of calculations, TMs are propagated by means of the rules of polynomial addition and multiplication or, whenever nonlinear operators occur, of Taylor expansion. The remainder is propagated by using both Taylor remainder and IA theory instead. Hence, TMs feature a rigorous and accurate parametric representation, while limiting the overestimation issue to the interval remainder only. The combination of the upper and lower bounds of the polynomial part with the remainder provides a guaranteed enclosure of the response. Applications of TMs are found in the solution of ODEs with bounded initial values [33]-[35].

This paper outlines, for the first time, an application of TMs to the parameterized modeling and WC analysis of linear circuits. From the uncertain design variables, a multivariate polynomial surface representing network responses is obtained and complemented by a conservative estimation of the approximation error. This representation yields both a parametric model of the response and the information on its WC bounds. The paper provides a self-contained description of the TM theory, together with the necessary extensions for its application to circuit simulations. In particular, specific solutions are proposed to improve the modeling accuracy and to extend the TM formulation to complex- and matrix-valued calculations, which are the key building blocks allowing to operate in the frequency domain. To the authors' best knowledge, an application of TMs to circuit analysis, with the aforementioned necessary extensions, has never been proposed in the literature.
The rest of the paper is organized as follows. The problem and the goals are stated in Section II. Section III briefly discusses the state-of-the-art interval methods, including IA which is used in the TM computations as described in Section IV. Section V addresses the extension of TMs to complexvalued matrix calculations, with specific emphasis on the matrix inversion. An illustrative as well as other application examples are provided as validations in Section VI. Section VII discusses the accuracy and the computational cost in comparison with alternative approaches. Finally, conclusions are drawn in Section VIII.

\section{Problem and Goal Statement}

This section lays the ground for the discussion on the proposed strategy for the parameterized and WC modeling of the frequency-domain response of a linear circuit with uncertain parameters. For the sake of illustration, the circuit of Fig. 1 is considered, in which the component values $R, L$ and $C$ are understood to be uncertain and defined within a bounded interval, rather than deterministically. This uncertainty on the circuit components produces a corresponding uncertainty on the voltage and current responses.

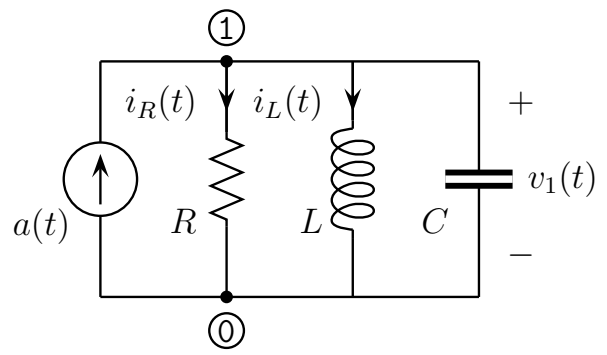

Fig. 1. Illustrative RLC circuit.

The frequency-domain solution for the sinusoidal steadystate response of the circuit of Fig. 1, computed via the modified nodal analysis (MNA) [36], reads

$$
\left[\begin{array}{l}
V_{1}(\omega) \\
I_{L}(\omega)
\end{array}\right]=\left[\begin{array}{cc}
G+j \omega C & 1 \\
1 & -j \omega L
\end{array}\right]^{-1}\left[\begin{array}{c}
A(\omega) \\
0
\end{array}\right],
$$

with $G=1 / R . V_{1}$ and $I_{L}$ are the phasors of the node voltage and inductor current, respectively, whereas $A$ denotes the phasor of the independent current source $a(t)$.

The first goal is to characterize the uncertainty due to the RLC components in terms of an upper and lower bound on $V_{1}$ and $I_{L}$. The second goal is to provide a parameterized model of these responses as function of the component values.

The standard approach to achieve the first goal is to perform a uniform MC sampling of the RLC values, to evaluate the circuit response (1) for each of these samples, and to calculate the aforementioned bounds as the minimum and maximum over the collected samples. This strategy, however, only provides an underestimation of the bounds, whose accuracy increases with the number of considered samples. Besides, the second goal is not achievable by the blind MC analysis.

The first task has been accomplished in a more effective way by using IA or AA computations [24]-[29], which 
produce conservative bounds for the calculations involved in (1). However, rather cumbersome solutions are required to improve the accuracy of the bound estimation, as discussed in the next section. Moreover, such techniques fail to provide a parameterized model.

In order to effectively achieve both goals, a specific tool is required that is capable of accurately propagating the uncertainty of system parameters in the calculation of (1), while keeping track of their interdependencies. Specifically, complex-valued operations like additions, multiplications and inversions, must be handled in both scalar and matrix forms. An effective solution is proposed and discussed in this paper based on the TM framework. In the following, the necessary theoretical notions are introduced step by step, starting from the definition of the scalar and real-valued interval calculations (Section III), then introducing the corresponding TM calculations (Section IV), and finally extending the framework to complex- and matrix-valued calculations (Section V).

\section{INTERVAL METHODS}

Interval methods are numerical techniques that replace the standard algebraic operations for bounded variables and produce a guaranteed enclosures of their result, by taking into account truncation and round-off errors in conservative way [23].

\section{A. Interval Arithmetic}

IA is based on the interval representation of uncertain parameters. The notation $\bar{x}=[a, b]$ is introduced to denote an interval variable that assumes values in the interval $[a, b]$. Given two IA-variables $\bar{x}=[a, b]$ and $\bar{y}=[c, d]$, it follows that

$$
\begin{aligned}
\bar{x}+\bar{y} & =[a+c, b+d] \\
\bar{x}-\bar{y} & =[a-d, b-c] \\
\bar{x} \cdot \bar{y} & =[\min (a c, a d, b c, b d), \max (a c, a d, b c, b d)] \\
\frac{\bar{x}}{\bar{y}} & =\left[\min \left(\frac{a}{c}, \frac{a}{d}, \frac{b}{c}, \frac{b}{d}\right), \max \left(\frac{a}{c}, \frac{a}{d}, \frac{b}{c}, \frac{b}{d}\right)\right],
\end{aligned}
$$

with the division being defined only as long as $0 \notin[c, d]$. The raise of an IA-variable to an integer power $k$ is

$$
\bar{x}^{k}= \begin{cases}{[1,1]} & k=0 \\ {\left[0, \max \left(a^{k}, b^{k}\right)\right]} & k \text { even, } 0 \in[a, b] \\ {\left[\min \left(a^{k}, b^{k}\right), \max \left(a^{k}, b^{k}\right)\right]} & \text { otherwise }\end{cases}
$$

The bounds resulting from the application of a nonlinear monotonic function $f(\cdot)$ to an IA-variable are readily given by

$$
f(\bar{x})=[\min (f(a), f(b)), \max (f(a), f(b))] .
$$

Despite being very simple to implement, the main limitation of IA is its inability to track any possible correlation between parameters, thus possibly leading to serious overestimation. As a typical example, the execution of the operation $\bar{x}-\bar{x}$ would produce $[a-b, b-a] \neq[0,0]$.

\section{B. Affine Arithmetic and Uncertainty Interval Partitioning}

$\mathrm{AA}$ is an alternative technique that overcomes the aforementioned limitation by analytically describing an interval variable in terms of a variation around its central value, i.e., $\tilde{x}=x_{0}+x_{1} \epsilon$, with $x_{0}=(a+b) / 2, x_{1}=(b-a) / 2$, and $\epsilon \in[-1,1]$. It follows immediately that $\tilde{x}-\tilde{x}=0$. Independent parameters are tracked by introducing a separate noise symbol. The AA form is in fact a linear parametric representation.

The uncertainty interval partitioning (UIP) is a strategy that subdivides the domain of each uncertain parameter into subintervals. The response bounds for each subdomain are computed separately via an interval method, and they are then combined to obtain the overall bounds. This approach improves the accuracy of the underlying interval method, although the number of partitions (and hence, of calculations) grows exponentially with the dimensionality. In [26], the UIP has been applied in conjunction with IA for WC circuit simulation.

AA and UIP may be used to improve the accuracy of TM-based calculations at the expense of the efficiency, as mentioned further. Nevertheless, they are not used in this paper and therefore not discussed in more detail. For additional information, the interested reader is referred to [23].

\section{TAYLOR MODELS}

This section provides a summary of the fundamental background of TMs, along with the key underlying steps involved in the basic operations and functional expansions in scalar form, for both the univariate and the multivariate cases. The extension to complex- and matrix-valued operations is discussed in the next section.

\section{A. Definition}

A TM is an hybrid representation of a nonlinear function of a bounded variable $x \in[a, b]$ in terms of a Taylor expansion plus an interval remainder. The $n$ th-order TM of $f(x)$ around the center of the interval $x_{0}$ is defined as

$$
T_{f}=P_{f}\left(x-x_{0}\right)+I_{f},
$$

where

$$
P_{f}\left(x-x_{0}\right)=\sum_{k=0}^{n} \frac{f^{(k)}\left(x_{0}\right)}{k !}\left(x-x_{0}\right)^{k}
$$

is the $n$ th-order Taylor expansion of $f(x)$ around $x_{0}$, whereas $I_{f}$ is an interval variable such that $f(x) \in P_{f}\left(x-x_{0}\right)+I_{f}$, $\forall x \in[a, b]$. Hence, the TM provides a parametric representation of $f(x)$ in terms of the polynomial $P_{f}$, while the remainder $I_{f}$ encloses the model between two curves within which lies the true value of the function. The bound of $f(x)$ is estimated as $B_{f}=B\left(P_{f}\right)+I_{f}$, where $B(\cdot)$ denotes the bound operator of the argument over its domain, and the addition is intended in the IA-sense.

The interval remainder $I_{f}$ is commonly defined as the bound of the Lagrange remainder of the Taylor expansion (9), which reads [38]

$$
R_{n}(x, \xi)=\frac{f^{(n+1)}(\xi)}{(n+1) !}\left(x-x_{0}\right)^{n+1},
$$


where $\xi \in\left[x_{0}, x\right]$. By increasing the order $n$, the remainder can be made arbitrarily small, thus improving the accuracy of the TM. Ideally, $I_{f}=[0,0]$ implies that $P_{f}$ provides an exact parametric representation of $f(x)$. The bound of the remainder (10) is estimated by means of IA-operations, starting from the interval representation of the variables $x$ and $\xi$.

It is relevant to point out that the calculation of the TM bounds requires the bounds of its polynomial part. In the univariate case, this calculation is trivial, as the bounds of a polynomial are merely the minimum and maximum over the values at the domain extrema and at the roots of its firstorder derivative that fall in the domain. Suitable solutions are required in the multivariate case instead, as discussed later on.

\section{B. Addition and Multiplication}

With the definitions provided, the addition and subtraction of TMs are straightforward, and lead to

$$
T_{f} \pm T_{g}=P_{f}\left(x-x_{0}\right) \pm P_{g}\left(x-x_{0}\right)+I_{f} \pm I_{g},
$$

where the operation over the remainders is carried out in the IA-sense.

The product between TMs yields instead

$$
\begin{aligned}
T_{u}= & T_{f} \cdot T_{g}=\left(P_{f}\left(x-x_{0}\right)+I_{f}\right)\left(P_{g}\left(x-x_{0}\right)+I_{g}\right) \\
& =P_{f}\left(x-x_{0}\right) P_{g}\left(x-x_{0}\right)+P_{f}\left(x-x_{0}\right) I_{g} \\
& +P_{g}\left(x-x_{0}\right) I_{f}+I_{f} I_{g} .
\end{aligned}
$$

The first term is expressed as

$$
P_{f}\left(x-x_{0}\right) P_{g}\left(x-x_{0}\right)=P_{u}\left(x-x_{0}\right)+P_{e}\left(x-x_{0}\right),
$$

where $P_{u}\left(x-x_{0}\right)$ is up to order $n$ and corresponds to the polynomial part of $T_{u}$, while $P_{e}\left(x-x_{0}\right)$ is the remaining higher-order contribution. The remainder of $T_{u}$ encompasses all remaining terms and is computed as

$$
I_{u}=B\left(P_{e}\right)+B\left(P_{f}\right) I_{g}+B\left(P_{g}\right) I_{f}+I_{f} I_{g},
$$

where the additions and multiplications are in the IA-sense.

\section{Functional Expansion}

The calculation of a nonlinear function $g(\cdot)$ of a TM $T_{f}$ is eased by considering the functional expansion w.r.t. the TM itself, rather than to the underlying variable $x$. Such an expansion is computed around the point $c_{f}=P_{f}\left(x_{0}\right)$, corresponding to the constant part of the TM. The TM $\bar{T}=T_{f}-c_{f}$, without constant part and defined by $\bar{P}_{f}\left(x-x_{0}\right)=P_{f}\left(x-x_{0}\right)-c_{f}$ and $\bar{I}_{f}=I_{f}$, is introduced. With the above definitions, the functional expansion reads

$$
\begin{aligned}
g\left(T_{f}\right) & =P_{g}\left(x-x_{0}\right)+I_{g} \\
= & \sum_{k=0}^{n} \frac{g^{(k)}\left(c_{f}\right)}{k !}\left(\bar{T}_{f}\right)^{k}+\frac{g^{(n+1)}(\varsigma)}{(n+1) !}\left(\bar{T}_{f}\right)^{n+1},
\end{aligned}
$$

where the derivatives of $g(\cdot)$ are computed w.r.t. its argument $T_{f}$ and with $\varsigma=c_{f}\left(1+[0,1] \cdot \bar{T}_{f} / c_{f}\right) \in B\left(T_{f}\right)$. It is worth noting that the first term in the r.h.s. of (15) merely involves sums and products of TMs, which are computed with the rules outlined in Section IV-B. Furthermore, since $\bar{T}_{f}$ has no constant part, the second term (Lagrange remainder) has degree $\geq n+1$, and therefore does not contribute to the polynomial part $P_{g}$. The bound of the Lagrange remainder is computed via IA-operations and it is added to the remainder of the first term to obtain the total remainder $I_{g}$. Alternatively, should $g(\cdot)$ be monotonic, the exact bound of $g\left(T_{f}\right)$ is determined from the bound of $T_{f}$ using (7), and a more accurate remainder is computed as $I_{g}=B\left(g\left(T_{f}\right)\right)-B\left(P_{g}\right)$.

\section{Multivariate Taylor Models}

The definition of a multivariate TM is straightforward. For the sake of convenience, the independent variables $\mathbf{x}=$ $\left[x_{1}, \ldots, x_{d}\right]$ are assumed to be normalized in the hyperdomain $D=[-1,1]^{d}$. This can always be achieved by proper shifting and rescaling. With this definition, retained throughout the rest of the paper, the center of the domain becomes $\mathbf{x}_{0}=0$, and the Taylor expansions reduce to Maclaurin series. The TM of a multivariate function $f(\mathbf{x})$ is expressed as $T_{f}=P_{f}(\mathbf{x})+I_{f}$ and encloses the true function between two hypersurfaces on $D$. The multivariate polynomial $P_{f}(\mathbf{x})$ collects the terms up to total degree $n$ (i.e., the monomials with degree $n$ or lower), while the remaining contributions are included in the interval remainder.

The definitions of addition and multiplication provided in Section IV-B also hold for the multivariate case. In the latter, the polynomial contribution of total degree higher than $n$ is collected into the extra polynomial $P_{e}$, whose bounds are included in the interval remainder. Moreover, the use of functional expansions as in Section IV-C eases the calculation of a nonlinear function of a multivariate TM, without the need for computing cumbersome partial derivatives.

When operating with multivariate TMs, the major issue comes from the determination of the bounds of the polynomial part, as needed for the determination of the WC as well as for the interval remainder of the multiplication (14) and functional expansion (15). This point is discussed in the next section.

\section{E. Multivariate Polynomial Bounds}

The calculation of the maximum and minimum of a multivariate polynomial on a bounded hyperdomain is a fundamental, yet non-trivial task in TM computations, which is often overlooked in the literature despite having a strong impact on the modeling accuracy. Several possible solutions are briefly reviewed in this section.

An exact solution exists for the bounds of a second-order polynomial. It is introduced and used in [20] to extract the bounds of a PC expansion. Nevertheless, the applicability of this approach is limited to first- and second-order TMs only; for the latter, a different strategy must still be devised for the bound of the extra polynomial $P_{e}$ arising in the multiplication.

The bound of a multivariate polynomial can be alternatively computed by using the interval definition of each uncertain design variable and by performing either IA or AA calculations on the monomials. The problem therefore reduces to the calculation of sums and products of interval variables. In 
particular, by separately considering the coefficient and the exponents of each monomial, the calculation for a univariate polynomial $P(x)=\sum_{i=0}^{n} p_{i} x^{i}$ in the normalized range $x=[-1,1]$ simplifies to

$$
p_{0}+\sum_{i=1}^{n} m_{i} \cdot\left|p_{i}\right| \leq P(x) \leq p_{0}+\sum_{i=1}^{n} M_{i} \cdot\left|p_{i}\right| .
$$

According to IA, the coefficients $M_{i}$ and $m_{i}$ are determined by the exponent $i$ and the sign of the coefficient $p_{i}$ as

$$
M_{i}= \begin{cases}0 & \text { if } p_{i}<0 \text { and } i \text { even } \\ 1 & \text { otherwise }\end{cases}
$$

and

$$
m_{i}= \begin{cases}0 & \text { if } p_{i}>0 \text { and } i \text { even } \\ -1 & \text { otherwise. }\end{cases}
$$

The generalization to the multivariate case is straightforward. In the following, this approach is referred to as the "raw" method. It is possibly combined with UIP to improve the accuracy of the estimation. This solution is suggested in [30] although, as already discussed, the number of operations does not scale favorably with the number of variables.

A more sophisticated strategy is proposed in this paper based on Bernstein polynomials. By means of a change of basis, a standard univariate polynomial $P(x)=\sum_{i=0}^{n} p_{i} x^{i}$ is converted into the Bernstein form

$$
P_{B}(x)=\sum_{i=0}^{n} b_{i} B_{i}(x),
$$

where the Bernstein basis polynomials are defined as

$$
B_{i}(x)=\left(\begin{array}{c}
n \\
i
\end{array}\right) x^{i}(1-x)^{n-i}, \quad 0 \leq i \leq n
$$

and the corresponding Bernstein coefficients are

$$
b_{i}(x)=\sum_{j=0}^{i} \frac{\left(\begin{array}{c}
i \\
j
\end{array}\right)}{\left(\begin{array}{c}
n \\
j
\end{array}\right)} 2^{j} \sum_{k=j}^{n}\left(\begin{array}{l}
k \\
j
\end{array}\right)(-1)^{k-j} p_{k} .
$$

Thanks to the properties of Bernstein polynomials [40][43], the polynomial $P_{B}(x)$ is contained within the interval spanned by the minimum and maximum Bernstein coefficients, i.e.,

$$
\min _{i}\left\{b_{i}\right\} \leq P(x) \leq \max _{i}\left\{b_{i}\right\} .
$$

Consider now a multivariate polynomial in the form

$$
P(\mathbf{x})=\sum_{\mathbf{k}} p_{\mathbf{k}} \prod_{j=1}^{d} x_{j}^{k_{j}},
$$

where $\mathbf{k}=\left[k_{1}, \ldots, k_{d}\right]$ is a multi-index defining the exponents of each monomial, with $0 \leq k_{j} \leq n$ and $\sum_{j=1}^{d} k_{j} \leq n$. Under the assumption that $\mathbf{x} \in[-1,1]^{d}$, the calculation of the vector of multivariate Bernstein coefficients simplifies to

$$
\mathbf{b}=\sum_{\mathbf{k}} p_{\mathbf{k}} \cdot\left(\boldsymbol{\beta}_{k_{1}} \otimes \boldsymbol{\beta}_{k_{2}} \otimes \cdots \otimes \boldsymbol{\beta}_{k_{d}}\right),
$$

where $\boldsymbol{\beta}_{k}$ is a vector with entries

$$
\beta_{k, i}=\sum_{j=0}^{\min \{i, k\}} \frac{\left(\begin{array}{l}
i \\
j
\end{array}\right)}{\left(\begin{array}{c}
n \\
j
\end{array}\right)} 2^{j}\left(\begin{array}{l}
k \\
j
\end{array}\right)(-1)^{k-j},
$$

for $i=0, \ldots, n$.

It is important to remark that the vectors $\boldsymbol{\beta}_{k}$, as needed for the conversion in (24), do not change and are therefore computed only once from $\boldsymbol{\beta}_{0}$ up to $\boldsymbol{\beta}_{n}$. For large variations of the input parameters, it may be necessary to introduce the UIP and divide the range into subintervals to avoid a detrimental loss of accuracy. However, the application examples proposed in the following show that Bernstein polynomials usually yield very high accuracy without the need for these subdivisions, whereas the "raw" method provides an easier and viable solution with an acceptable accuracy.

\section{COMPleX AND MATRIX OPERATIONS}

The notions introduced so far allow to perform scalar and real-valued calculations. In order to deal with frequencydomain circuit equations like (1), an extension of the TM tool to complex-valued matrix computations is necessary. This fundamental step is discussed in this section, with specific emphasis on the matrix inversion. It is worth noting that calculating the inverse of a matrix TM with a guaranteed conservative bound is a non-trivial task that, to the authors' best knowledge, has never been documented in the literature before.

\section{A. Complex Calculus}

The extension to complex algebra is relatively straightforward and is carried out based on the fundamental rules. Specifically, given two scalar and complex variables in rectangular form:

$$
\begin{aligned}
& T_{f}=T_{f, r}+j T_{f, i} \\
& T_{g}=T_{g, r}+j T_{g, i}
\end{aligned}
$$

with their real and imaginary parts defined as TMs like (8), the sum and subtraction reduce to $T_{f} \pm T_{g}=\left(T_{f, r} \pm T_{g, r}\right)+$ $j\left(T_{f, i} \pm T_{g, i}\right)$. The multiplication and division become instead

$$
T_{f} \cdot T_{g}=\left(T_{f, r} T_{g, r}-T_{f i} T_{g, i}\right)+j\left(T_{g, r} T_{f, i}+T_{g, i} T_{f, r}\right)
$$

and

$$
T_{f} / T_{g}=\frac{T_{f, r} T_{g, r}+T_{f, i} T_{g, i}}{T_{g, r}^{2}+T_{g, i}^{2}}+j \frac{T_{f, i} T_{g, r}-T_{g, i} T_{f, r}}{T_{g, r}^{2}+T_{g, i}^{2}},
$$

respectively. The real and imaginary parts are therefore handled separately by means of the real-valued computations discussed in Section IV.

The calculation of the magnitude in linear scale

$$
\left|T_{f}\right|=\sqrt{T_{f, r}^{2}+T_{f, i}^{2}}
$$

or in $\mathrm{dB}$

$$
\left|T_{f}\right|_{\mathrm{dB}}=10 \log \left(T_{f, r}^{2}+T_{f, i}^{2}\right)
$$

is performed by applying the rules of function expansion (15) to the square-root and logarithmic functions. 


\section{B. Matrix Taylor Models and Matrix Inversion}

A matrix TM is denoted as $\boldsymbol{T}_{f}=\boldsymbol{P}_{f}(\mathbf{x})+\boldsymbol{I}_{f}$, where each quantity is understood to be a matrix with entries consistent with the standard TM representation (8). The basic scalar operations introduced so far, i.e., addition, subtraction and multiplication, are readily generalized to the corresponding matrix calculations by operating element-wise. In particular, the product of two matrix TMs merely involves additions and multiplications of scalar TMs.

The matrix inversion requires a suitable algorithm instead. An effective approach based on the Sherman-Morrison (SM) formula [39] is proposed in the following. The SM formula provides a clever and exact solution for the computation of the inversion $(\boldsymbol{A}+\boldsymbol{B})^{-1}$ when $\boldsymbol{B}$ has unitary rank. In this case, the resulting inverse matrix is

$$
(\boldsymbol{A}+\boldsymbol{B})^{-1}=\boldsymbol{A}^{-1}-\frac{1}{1+\alpha}\left(\boldsymbol{A}^{-1} \boldsymbol{B} \boldsymbol{A}^{-1}\right),
$$

where $\alpha=\operatorname{tr}\left(\boldsymbol{A}^{-1} \boldsymbol{B}\right)$. Hence, the inversion of the sole matrix $\boldsymbol{A}$ is actually required.

Consider now a matrix $\mathrm{TM} \boldsymbol{T}_{f}$, which is conveniently split into the sum of a constant matrix and a shifted TM, as was done in Section IV-C for the scalar case:

$$
\boldsymbol{T}_{f}=\boldsymbol{c}_{f}+\left[\overline{\boldsymbol{P}}_{f}(\mathbf{x})+\boldsymbol{I}_{f}\right]=\boldsymbol{c}_{f}+\overline{\boldsymbol{T}}_{f}
$$

where $\boldsymbol{c}_{f}=\boldsymbol{P}_{f}(0)$ and $\overline{\boldsymbol{P}}_{f}(\mathrm{x})=\boldsymbol{P}_{f}(\mathrm{x})-\boldsymbol{c}_{f}$. If the nonconstant part $\overline{\boldsymbol{T}}_{f}$ has unitary rank, then (33) is applied directly and the inverse $\mathrm{TM}$ is

$$
\begin{aligned}
\boldsymbol{T}_{g} & =\boldsymbol{T}_{f}^{-1}=\left(\boldsymbol{c}_{f}+\overline{\boldsymbol{T}}_{f}\right)^{-1} \\
& =\boldsymbol{c}_{f}^{-1}-\frac{1}{1+\alpha}\left(\boldsymbol{c}_{f}^{-1} \overline{\boldsymbol{T}}_{f} \boldsymbol{c}_{f}^{-1}\right),
\end{aligned}
$$

with $\alpha=\operatorname{trace}\left(\boldsymbol{c}_{f}^{-1} \overline{\boldsymbol{T}}_{f}\right)$. It is relevant to remark that the SM formula allows to reduce the inversion of a matrix TM to standard and already available calculations, namely additions and multiplications of TMs, the inversion of the constant matrix $\boldsymbol{c}_{f}$, and the inversion of the scalar TM of $1+\alpha$. In practical situations, the matrix $\boldsymbol{c}_{f}$ has always full rank, as it corresponds to the matrix for the nominal design. Moreover, the trace operation merely involves a sum of TMs.

The generalization of (33) to the case of full-rank matrices requires an iterative application of the rule, by splitting the matrix $\overline{\boldsymbol{T}}_{f}$ into the sum of rank-one matrices:

$$
\overline{\boldsymbol{T}}_{f}=\overline{\boldsymbol{T}}_{f, 1}+\overline{\boldsymbol{T}}_{f, 2}+\overline{\boldsymbol{T}}_{f, 3}+\ldots,
$$

where the notation $\overline{\boldsymbol{T}}_{f, i}$ denotes a matrix $\mathrm{TM}$ with null entries except for the $i$ th column, which coincides with the $i$ th column of the original TM $\overline{\boldsymbol{T}}_{f}$. Hence, (31) is first used to compute the inversion $\left(\boldsymbol{c}_{f}+\overline{\boldsymbol{T}}_{f, 1}\right)^{-1}$, by assuming $\boldsymbol{A}=\boldsymbol{c}_{f}$ and $\boldsymbol{B}=\overline{\boldsymbol{T}}_{f, 1}$. In the second step, the inversion $\left(\boldsymbol{c}_{f}+\overline{\boldsymbol{T}}_{f, 1}+\overline{\boldsymbol{T}}_{f, 2}\right)^{-1}$ is calculated by letting $\boldsymbol{A}=\boldsymbol{c}_{f}+\overline{\boldsymbol{T}}_{f, 1}$ and $\boldsymbol{B}=\overline{\boldsymbol{T}}_{f, 2}$. The inverse of $\boldsymbol{A}$, as needed in (31), is available from the previous step. The procedure is iterated until all terms in (34) are accounted for, thus yielding the end result.

\section{APPLICATION EXAMPLES}

The proposed technique has been implemented in MATLAB on an Apple MacBook Pro with an Intel(R) i5, CPU running at $2.4 \mathrm{GHz}$ and $4 \mathrm{~GB}$ of RAM. In the following, it is applied to the frequency-domain parametric and WC simulation of several circuits with bounded uncertain parameters.

\section{A. Illustrative Example (RLC Circuit)}

As an illustrative example, the RLC circuit of Fig. 1 is considered first, with focus on the network function $H(\omega)=$ $I_{L}(\omega) / A(\omega)$ and on the effects of the parameters $G$ and $L$ on the behavior. The above quantities have bounded uncertainties with $10 \%$ and $25 \%$ maximum relative variations around their nominal values, i.e., $G=[0.9,1.1] \mathrm{S}$ and $L=[1.5,2.5] \mathrm{H}$, whereas the capacitance has a fixed value of $C=1 \mathrm{~F}$. The uncertain design parameters are represented by the zerocentered TMs

$$
\begin{aligned}
& T_{G}(\mathbf{x})=1+0.1 x_{1}+[0,0] \\
& T_{L}(\mathbf{x})=2+0.5 x_{2}+[0,0]
\end{aligned}
$$

with $\mathbf{x}=\left[x_{1}, x_{2}\right] \in[-1,1] \times[-1,1]$. The above TMs replace the conductance and inductance in (1), thus yielding the following TM of the MNA matrix

$$
\boldsymbol{M}(\mathbf{x})=\left[\begin{array}{cc}
1+j \omega & 1 \\
1 & -j 2 \omega
\end{array}\right]+\left[\begin{array}{cc}
0.1 x_{1} & 0 \\
0 & -j 0.5 \omega x_{2}
\end{array}\right]
$$

with null interval remainders. From (1), the corresponding TM of the transfer function $H(\omega, G, L)$, let us denote it as $T_{H}=$ $P_{H}(\omega, G, L)+I_{H}(\omega)$, is provided by the element $\left[\boldsymbol{M}^{-1}\right]_{21}$ of the inverse of matrix (37), which is calculated by applying the algebraic rules outlined in Sections IV and V. The Appendix provides the interest reader with the step-by-step calculation of this inversion.
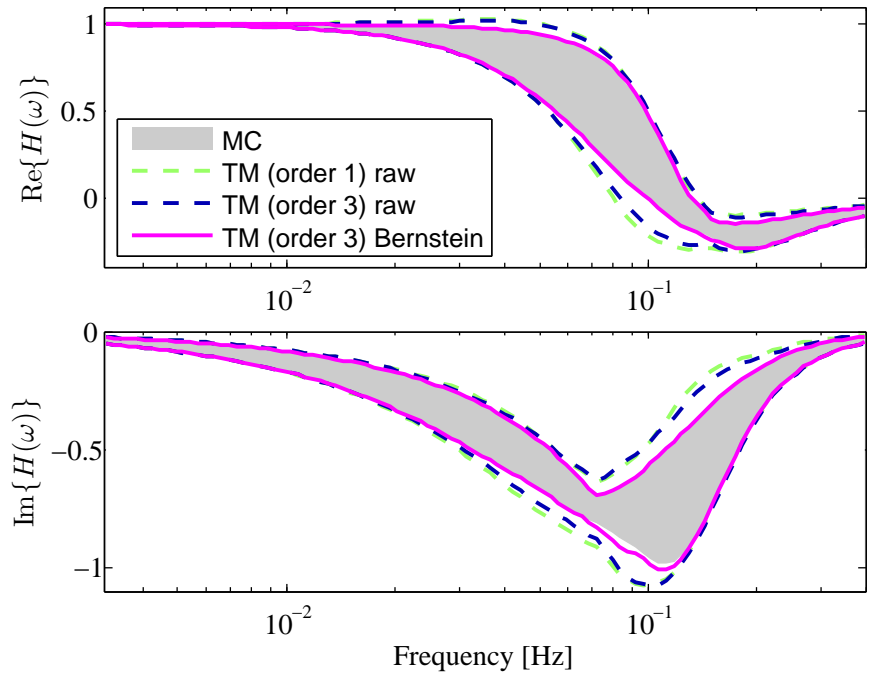

Fig. 2. Transfer function $H(j \omega)=I_{L}(\omega) / A(\omega)$ in the lumped circuit of Fig. 1. The spread of the response is provided by the superposition of many MC samples (gray area). The WC bounds are obtained with first- and thirdorder TMs and the "raw" calculation of the polynomial bounds (dashed lines), or with a third-order TM and Bernstein polynomials (solid lines).

Fig. 2 shows the WC bounds of the real and imaginary parts of $H(\omega)$ over 100 frequency points, computed by means of 
the proposed technique as $B\left(P_{H}\right)(\omega)+I_{H}(\omega)$ and compared with the spread given by the superposition of $10000 \mathrm{MC}$ responses (gray area). The TM results are produced by considering different expansion orders and/or different approaches to evaluate the upper and lower bounds of the polynomial part. Specifically, the dashed lines refer to a first- or thirdorder expansion with the "raw" calculation of the polynomial bounds. The solid line refers to a third-order expansion with Bernstein polynomials instead. The calculation via Bernstein polynomials yields a substantial improvement in the estimation of the WC, without resorting to the UIP. This example also shows that the accuracy in the determination of the polynomial bounds (which is, in general, an open issue for the multivariate case) plays a crucial role.
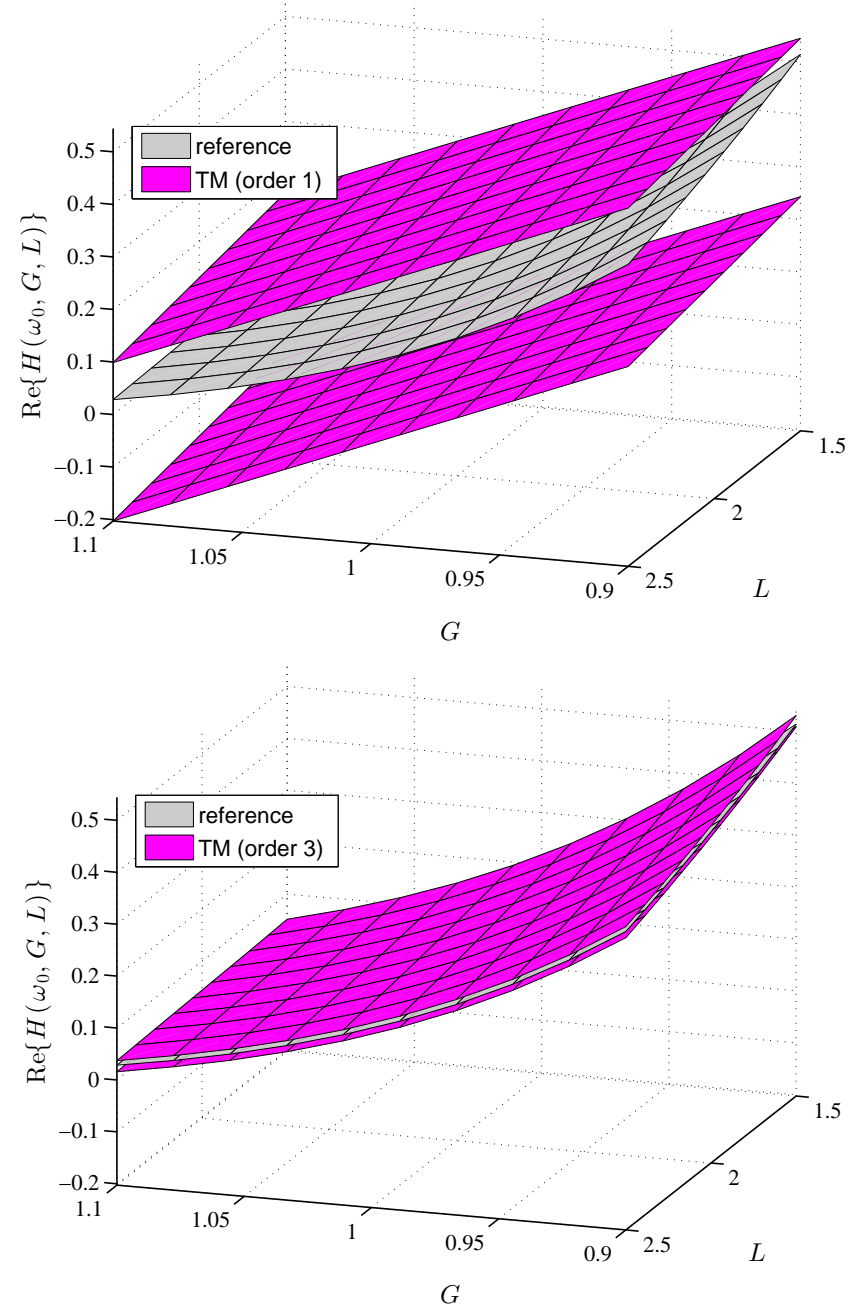

Fig. 3. Real part of the transfer function $H(\omega)$ at $\omega=0.61 \mathrm{rad} / \mathrm{s}$ plotted as a function of $G$ and $L$. The gray surface is the exact solution. The colored surfaces are the upper and lower bounds given by the first- (top panel) and third-order (bottom panel) TMs.

To further highlight the features of the TM approach, Fig. 3 plots the real part of the complete two-dimensional TM $P_{H}\left(\omega_{0}, G, L\right)+I_{H}\left(\omega_{0}\right)$ (colored surfaces) as a function of $G$ and $L$ at the angular frequency $\omega_{0}=0.61 \mathrm{rad} / \mathrm{s}$. The actual response (gray surface) is guaranteed to lie within these two bounds and, as shown, this is indeed the case. The results are provided for first- and third-order TMs (top and bottom panels, respectively). As expected, the first-order TM defines two planes, with a rough estimation of the maximum and minimum of the circuit response within the given domain. The thirdorder TM defines a cubic surface instead, with an improved accuracy established by the tighter bounding provided by the colored surfaces. These results highlight the capability of the TM to provide a tight bound for the response variation as the order is increased. Moreover, the truncation of the Taylor expansion is suitably accounted for by the interval remainder, leading to a conservative upper and lower bounding surfaces.

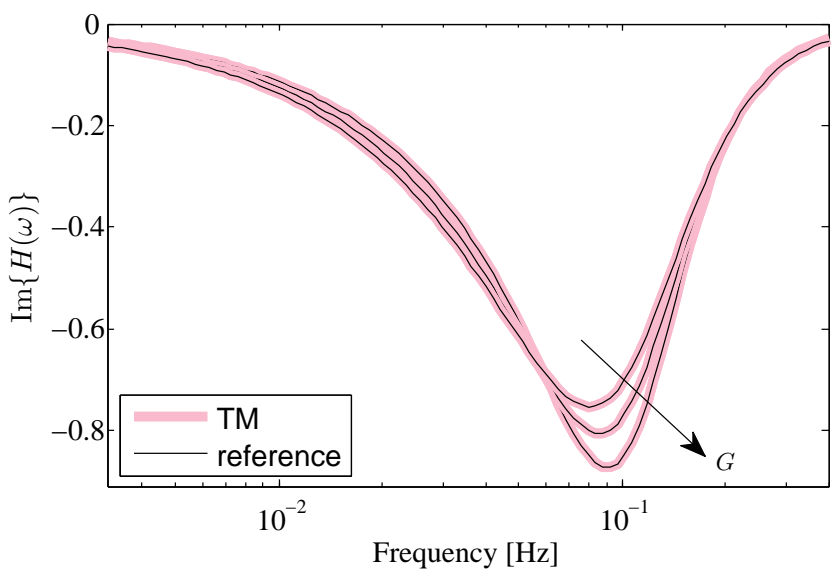

Fig. 4. Imaginary part of $H(\omega)$ for varying values of the conductance $G$. The reference responses (thin black curves) are compared against the parameterized model provided by a third-order TM (thick colored curves).

Finally, Fig. 4 shows the parametric response of the imaginary part of $H\left(\omega, G, L_{0}\right)$, as a function of the conductance $G$ and with the inductance value fixed to the nominal value $L_{0}=2 \mathrm{H}$. The reference responses (thin black curves), obtained by sweeping the value of $G$ from $0.9 \mathrm{~S}$ to $1.1 \mathrm{~S}$, are compared with the parametric model provided by the polynomial part $P_{H}\left(\omega, G, L_{0}\right)$ of the third-order TM (colored thick lines), showing excellent agreement.

\section{B. Active Low-Pass Filter}

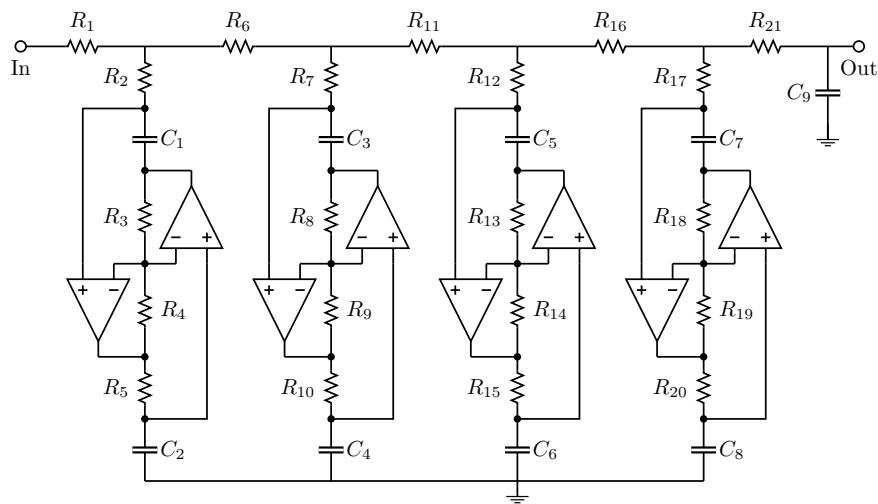
$\begin{array}{lllll}R_{1}=5.4779 \mathrm{k} \Omega & R_{2}=2.0076 \mathrm{k} \Omega & R_{3,4,8,9,13,14,18,19}=3.3 \mathrm{k} \Omega & R_{5}=4.5898 \mathrm{k} \Omega \\ R_{6}=4.44 \mathrm{k} \Omega & R_{7}=5.9999 \mathrm{k} \Omega & R_{10}=4.2573 \mathrm{k} \Omega & R_{11}=3.2201 \mathrm{k} \Omega & R_{12}=5.88327 \mathrm{k} \Omega\end{array}$ $\begin{array}{lllll}R_{15}=5.62599 \mathrm{k} \Omega & R_{16}=3.63678 \mathrm{k} \Omega & R_{17}=1.0301 \mathrm{k} \Omega & R_{20}=5.808498 \mathrm{k} \Omega & R_{21}=1.2201 \mathrm{k} \Omega \\ C_{1}=12 \mathrm{nF} & C_{3}=6.8 \mathrm{nF} & C_{5}=4.7 \mathrm{nF} & C_{7}=6.8 \mathrm{nF} & C_{2,4,6,8,9}=10 \mathrm{nF}\end{array}$

Fig. 5. Schematic of the active low-pass filter (reproduced from [19]). 
The proposed technique is now applied to the analysis of a more complex network, i.e., the active low-pass filter [19] shown in Fig. 5. The operational amplifiers are considered to be ideal. The nominal values of all circuit parameters are provided in Fig. 5. Additionally, five parameters, namely $C_{2}, C_{4}, C_{6}, C_{8}$, and $C_{9}$, exhibit a variation within $\pm 5 \%$ around their nominal values. The variations of pairs $C_{2}-C_{4}$ and $C_{6}-C_{8}$ are correlated and described by means of one independent variable each. Together with $C_{9}$, the total number of independent variables amounts to three.
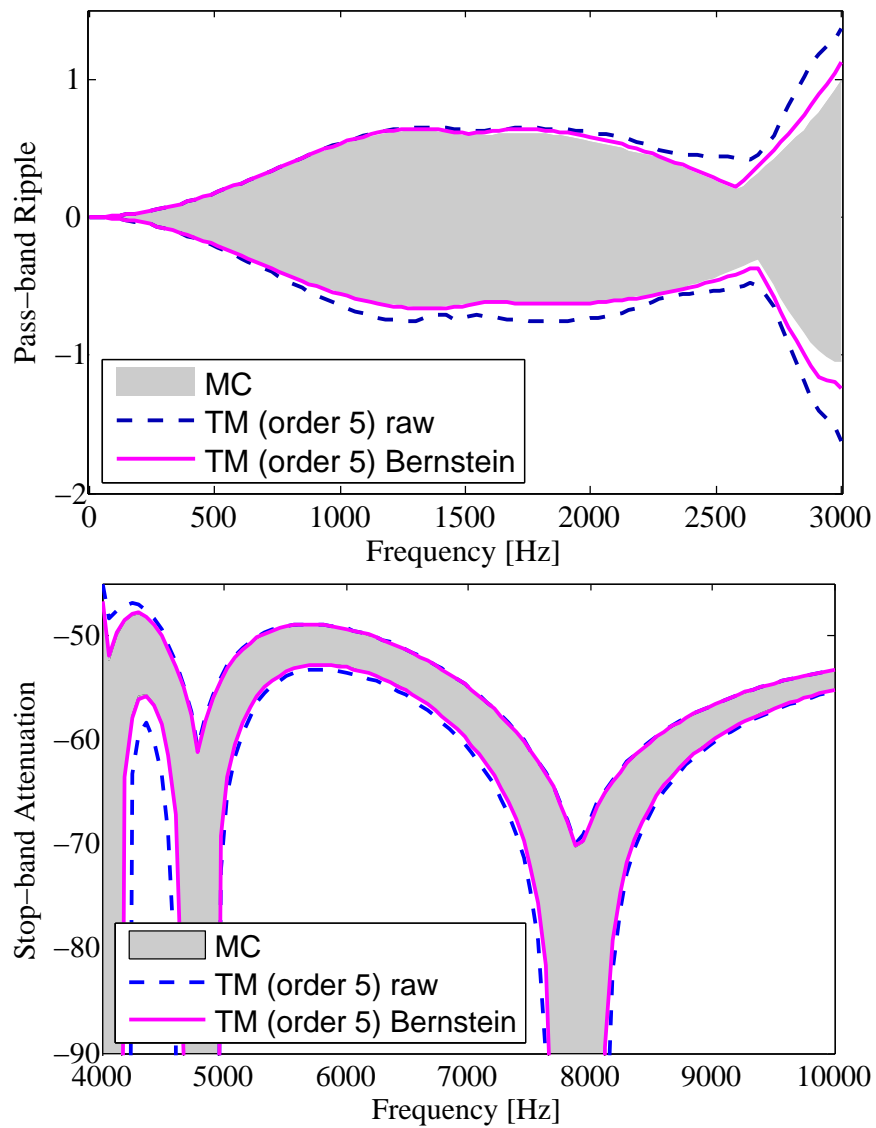

Fig. 6. Passband ripple (top panel) and stopband attenuation (bottom panel) of the active filter of Fig. 5. The upper and lower WC bounds obtained with a fifth-order TM and the raw (dashed lines) or Bernstein (solid lines) approaches are compared against the spread of the MC samples (gray area).

The magnitude of the transfer function $H(\omega)=$ $V_{\text {out }}(\omega) / V_{\text {in }}(\omega)$ is provided in Fig. 6 for both the passband (top panel), extending from 0 to $3000 \mathrm{~Hz}$, and the stopband (bottom panel), starting at $4000 \mathrm{~Hz}$ and exhibiting a nominal minimum attenuation of $50 \mathrm{~dB}$. The $\mathrm{WC}$ bounds provided by a fifth-order TM and computed with both the raw (dashed line) and Bernstein (solid line) approaches are compared against the spread of $10000 \mathrm{MC}$ samples. The comparison confirms once again the superior accuracy of the Bernstein approach, but also the reasonably good estimation achieved with the much simpler raw method.

Furthermore, Fig. 7 compares a small subset of MC responses (thin black lines) against the parametric model provided by the TM (thick colored lines). An excellent agreement is established, in spite of the large variation of the responses.
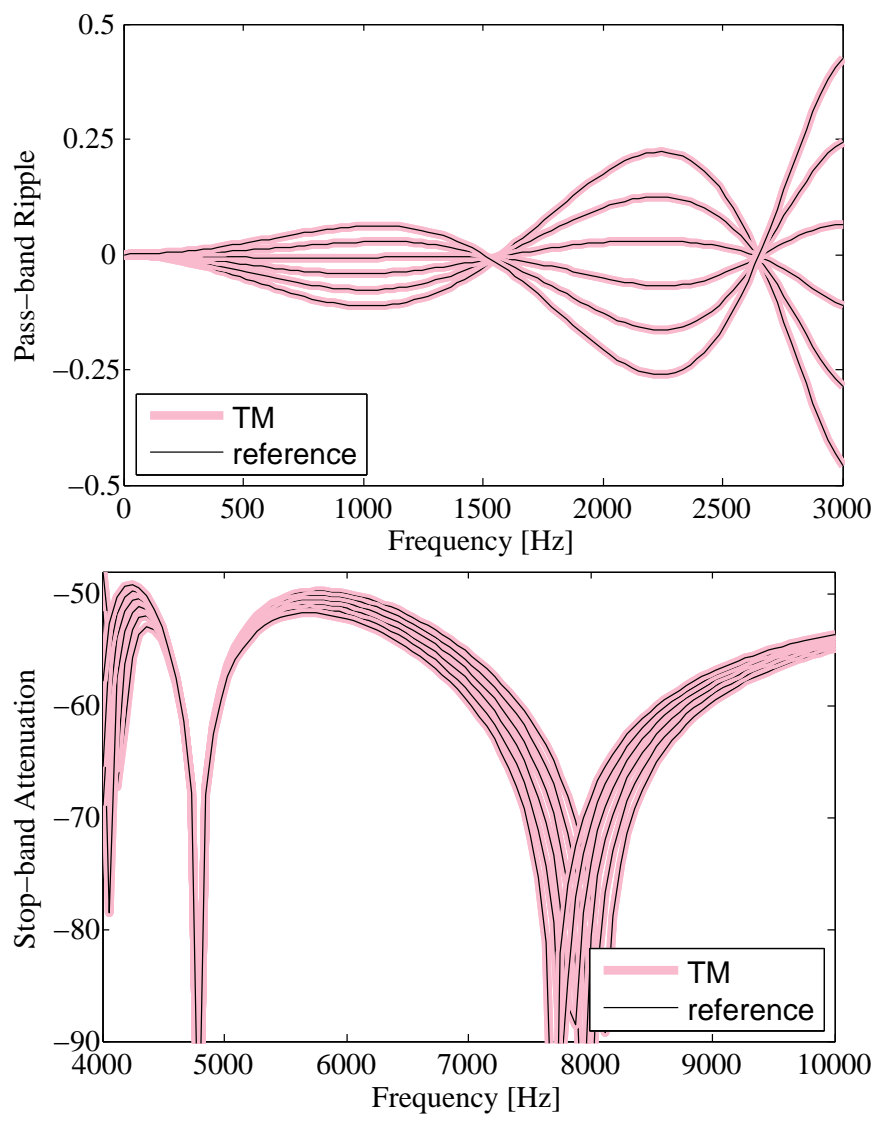

Fig. 7. Passband (top panel) and stopband (bottom panel) responses for different values of the uncertain design parameters. The reference responses (thin black curves) are compared against the TM prediction (thick colored lines).

\section{RF Filter}

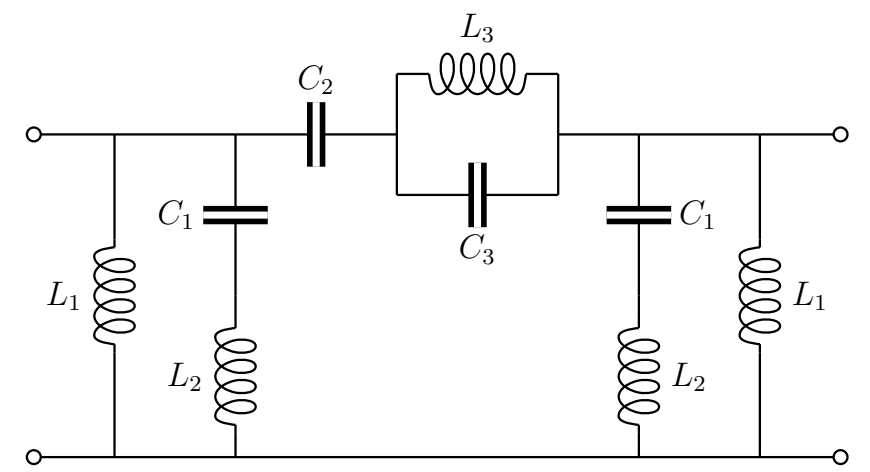

Fig. 8. Schematic of the band- and high-pass Chebyshev filter.

The last example refers to the two-port RF filter shown in Fig. 8. The filter is of Chebyshev type and it is designed to exhibit both a band- and high-pass behavior. The nominal values of the circuit components are: $L_{1}=146 \mathrm{nH}, L_{2}=$ $312.5 \mathrm{nH}, L_{3}=92 \mathrm{nH}, C_{1}=73.6 \mathrm{pF}, C_{2}=116.6 \mathrm{pF}, C_{3}=$ $250 \mathrm{pF}$. The inductances $L_{1}, L_{2}$ and $L_{3}$ are considered as 3 independent parameters that exhibit a maximum variation of $\pm 5 \%$ around their nominal values. The ports have a reference impedance of $50 \Omega$. 


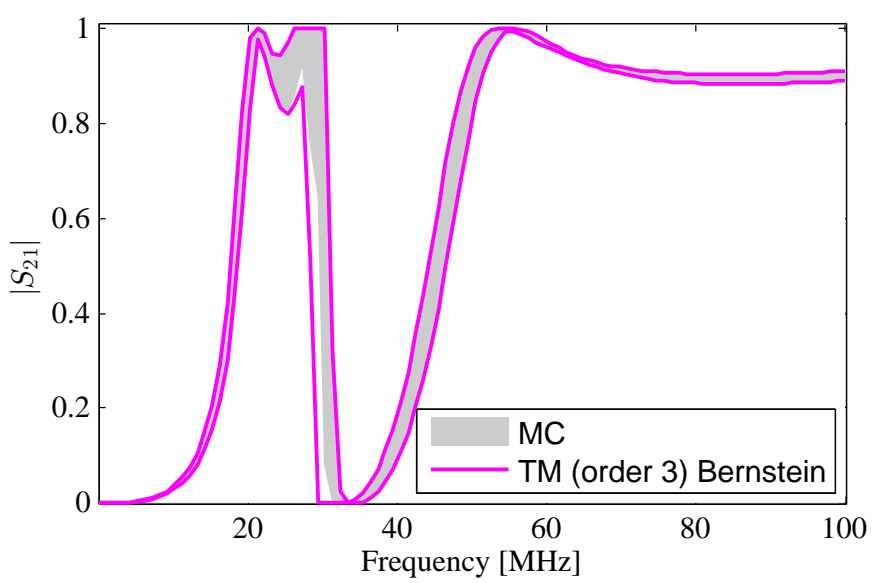

Fig. 9. Magnitude of $S_{21}$ in the filter of Fig. 8. The response variation (gray area) is compared against the upper and lower WC bounds estimated via a third-order TM in conjunction with the Bernstein approach (solid thick lines).

Fig. 9 shows the magnitude of the scattering parameter $S_{21}$. The spread due to the parameter variations and computed via the superposition of $10000 \mathrm{MC}$ samples (gray area) is compared against the WC bounds estimated via a third-order TM (solid lines). The Bernstein approach is used for the bounds of the polynomial part. For a more accurate calculation, the physical constraints $\left|S_{21}\right| \leq 1$ and $-1 \leq \operatorname{Re}\left\{S_{21}\right\}, \operatorname{Im}\left\{S_{21}\right\} \leq 1$ on the scattering parameters are taken into account. The results once again confirm the accuracy of the proposed TM method in predicting the WC bounds.

\section{PERFormance AND COMPUTATIONAL COST}

This section discusses the performance of the proposed simulation framework in estimating WC responses in comparison with classical techniques, and the corresponding computational cost.

\section{A. Convergence of MC Analysis and TM Accuracy}

The numerical analysis of the accuracy is focused on a selection of frequencies from the results in Fig. 6 and Fig. 9. Table I collects the WC bounds of the response of the active and RF filters obtained with MC, the proposed TM approach in conjunction with Bernstein polynomials, and a PC expansion with exact bound calculation [20].

The first four rows of Table I provide the results obtained with different sample sizes in the MC analysis and disclose a rather slow convergence rate. The difference between the results for $10^{5}$ and $10^{6}$ samples is, in some cases, still relatively large. This is explained by the fact that WC responses may lie in a region with lower probability density and have therefore a low chance to be approached. As for the TM result (fifth row), it always provides a conservative estimation, which is normally very close to the MC result with the largest number of samples. On the other hand, the table also shows that the PC expansion often underestimates the MC result. This is because the approximation error introduced by the PC expansion is defined in statistical terms and does not prevent an underestimation of the response.
It is relevant to point out that the "true" WC bounds lie between the results from MC (inherently underestimated) and TM (guaranteed conservative) analyses, which can be therefore regarded as complementary tools, in analogy with the combined use of $\mathrm{MC}$ and $\mathrm{AA}$ in [27]. As for $\mathrm{PC}$, it is impossible to predict a priori whether overestimation or underestimation is provided.

\section{B. Computational Cost}

TABLE II

COMPUTATIONAL COST PER FREQUENCY POINT FOR THE PROPOSED TM APPROACH IN THE DIFFERENT APPLICATION EXAMPLES.

\begin{tabular}{|l|r|r|r|}
\hline example & TM (raw) & TM (Bernstein) & equivalent MC runs \\
\hline \hline RLC circuit $(d=1)$ & $0.11 \mathrm{~s}$ & $0.12 \mathrm{~s}$ & 1100 \\
RLC circuit $(d=2)$ & $0.11 \mathrm{~s}$ & $0.13 \mathrm{~s}$ & 1200 \\
\hline active filter $(d=1)$ & $8.31 \mathrm{~s}$ & $8.96 \mathrm{~s}$ & 74700 \\
active filter $(d=2)$ & $12.55 \mathrm{~s}$ & $14.41 \mathrm{~s}$ & 120100 \\
active filter $(d=3)$ & $14.05 \mathrm{~s}$ & $22.40 \mathrm{~s}$ & 186700 \\
\hline RF filter $(d=1)$ & $2.29 \mathrm{~s}$ & $2.45 \mathrm{~s}$ & 30700 \\
RF filter $(d=2)$ & $2.74 \mathrm{~s}$ & $3.01 \mathrm{~s}$ & 37700 \\
RF filter $(d=3)$ & $3.46 \mathrm{~s}$ & $4.45 \mathrm{~s}$ & 55700 \\
\hline
\end{tabular}

Table II collects the simulation times of the TM calculations for the three application examples included in this paper. In order to quantify the overhead of using Bernstein polynomials in place of the raw bound estimation, both cases are reported in the table. The impact of the problem dimensionality is also assessed by varying the dimension $d$ of the design parameter space. The table shows that the method scales relatively well with the dimension of the parameter space and that the overhead in the use of Bernstein polynomials is generally limited, except for larger problems in terms of network size and number of design parameters. Moreover, the last column indicates the number of samples that can be obtained in the same amount of time with a classical MC analysis. In most of the cases, the value is well below $10^{5}$, which may result in an inaccurate estimation of the WC bounds, as shown in Table I, and without the additional insight provided by the parametric model. It should be noted that the margin for improvement by mere implementational optimization of the TM operations is rather large, whereas the primary goal of this paper is to provide a proof of concept of the method's validity and applicability.

\section{CONCLUSIONS}

This paper presents a TM-based approach to jointly obtain a parameterized frequency-domain response of a circuit with bounded uncertain parameters and the corresponding WC bounds. A TM consists of a Taylor polynomial expansion, which represents the parametric model, and of an interval reminder, which accounts for approximation errors in a conservative way. The combination of the upper and lower bounds of the polynomial part with the interval remainder provides a conservative estimation of the $\mathrm{WC}$ response. 
TABLE I

COMPARISON ON THE ACCURACY BETWEEN MC, TM AND PC IN THE ESTIMATION OF THE WC BOUNDS OF THE ACTIVE AND RF FILTER RESPONSES.

\begin{tabular}{|c|c|c|c|c|c|c|}
\hline \multirow[b]{2}{*}{$\downarrow$ method / frequency $\rightarrow$} & \multicolumn{3}{|c|}{ active low-pass filter } & \multicolumn{3}{|c|}{ RF filter } \\
\hline & $4200 \mathrm{~Hz}$ & $5000 \mathrm{~Hz}$ & $8200 \mathrm{~Hz}$ & $23 \mathrm{MHz}$ & $52 \mathrm{MHz}$ & $53 \mathrm{MHz}$ \\
\hline MC (10 3 samples $)$ & {$[-60.2162,-48.4460]$} & {$[-65.5689,-53.8135]$} & {$[-77.2765,-63.7759]$} & {$[0.9014,0.9524]$} & {$[0.9408,0.9893]$} & {$[0.9725,0.9980]$} \\
\hline MC (10 5 samples $)$ & {$[-60.7607,-48.3115]$} & {$[-65.8212,-53.6614]$} & {$[-77.4607,-63.7334]$} & {$[0.8992,0.9528]$} & {$[0.9329,0.9908]$} & {$[0.9675,0.9986]$} \\
\hline MC (10 6 samples $)$ & {$[-60.8430,-48.2923]$} & {$[-65.8212,-53.6495]$} & {$[-77.5000,-63.7205]$} & {$[0.8990,0.9532]$} & {$[0.9326,0.9908]$} & {$[0.9673,0.9986]$} \\
\hline PC & {$[-60.1618,-48.5002]$} & {$[-65.2900,-53.8358]$} & {$[-76.5687,-63.9950]$} & {$[0.8992,0.9531]$} & {$[0.9330,0.9900]$} & {$[0.9679,0.9977]$} \\
\hline
\end{tabular}

The technique is applied to the frequency-domain circuit analysis through a suitable re-definition of the algebraic operations involved in the calculation of the output responses from the (possibly uncertain) circuit parameters. Original and novel solutions are proposed to extend the TM framework to matrix and complex-valued calculations, and to improve the estimation of the WC bounds. Specifically, an iterative approach based on the SM formula is put forward to invert a matrix TM. Moreover, the conversion of the Taylor expansion into Bernstein polynomials is proposed to improve the estimation of the upper and lower bounds.

Several application examples are provided to demonstrate the accuracy and the feasibility of the technique. It is also highlighted that the accuracy of the WC bounds strongly depends on the accurate calculation of the maximum and minimum of the polynomial part of the TM. However, the use of Bernstein polynomials allows to achieve a substantial improvement with respect to traditional solutions based, e.g., on IA calculations, while avoiding the use of the UIP. The methodology offers promising accuracy and competitive computational efficiency in comparison with standard techniques.

\section{ACKNOWLEDGMENTS}

The authors would like to thank Prof. Hendrik Rogier, Ghent University, for providing the design of the RF filter, and to acknowledge Prof. C. K. Michael Tse, Editor-in-Chief of the IEEE Circuits and Systems Magazine, for stimulating their interest on Taylor models.

\section{APPENDIX A \\ INVERSION OF MATRIX TMS}

This appendix outlines the step-by-step inversion of the matrix TM (37) via the SM algorithm. To begin with, the TM is rewritten as $\boldsymbol{M}(\mathbf{x})=\boldsymbol{c}_{M}+\overline{\boldsymbol{T}}_{M, 1}+\overline{\boldsymbol{T}}_{M, 2}$, with

$$
\begin{aligned}
\boldsymbol{c}_{M} & =\left[\begin{array}{cc}
1+j \omega & 1 \\
1 & -j 2 \omega
\end{array}\right] \\
\overline{\boldsymbol{T}}_{M, 1} & =\left[\begin{array}{cc}
0.1 x_{1} & 0 \\
0 & 0
\end{array}\right] \\
\overline{\boldsymbol{T}}_{M, 2} & =\left[\begin{array}{cc}
0 & 0 \\
0 & -j 0.5 \omega x_{2}
\end{array}\right]
\end{aligned}
$$

The application of the SM algorithm requires the preliminary calculation of the inverse of $\boldsymbol{c}_{M}$. By focusing on the angular frequency $\omega=0.61 \mathrm{rad} / \mathrm{s}$, as for the results in Fig. 3, the inverse reads

$$
\boldsymbol{c}_{M}^{-1}=\left[\begin{array}{rr}
0.958+j 0.201 & 0.165-j 0.785 \\
0.165-j 0.785 & -0.644+j 0.685
\end{array}\right]
$$

The first step is then the calculation of $\left(\boldsymbol{c}_{M}+\overline{\boldsymbol{T}}_{M, 1}\right)^{-1}$ as

$$
\left(\boldsymbol{c}_{M}+\overline{\boldsymbol{T}}_{M, 1}\right)^{-1}=\boldsymbol{c}_{M}^{-1}-\frac{1}{1+\operatorname{tr}\left(\boldsymbol{c}_{M}^{-1} \overline{\boldsymbol{T}}_{M, 1}\right)}\left(\boldsymbol{c}_{M}^{-1} \overline{\boldsymbol{T}}_{M, 1} \boldsymbol{c}_{M}^{-1}\right)
$$

The partial calculations involved are as follows:

$$
\begin{gathered}
\boldsymbol{c}_{M}^{-1} \overline{\boldsymbol{T}}_{M, 1}=\left[\begin{array}{ll}
0.096 x_{1} & 0 \\
0.016 x_{1} & 0
\end{array}\right]+j\left[\begin{array}{rr}
0.020 x_{1} & 0 \\
-0.079 x_{1} & 0
\end{array}\right] \\
\operatorname{tr}\left(\boldsymbol{c}_{M}^{-1} \overline{\boldsymbol{T}}_{M, 1}\right)=0.096 x_{1}+j 0.020 x_{1} \\
\boldsymbol{c}_{M}^{-1} \overline{\boldsymbol{T}}_{M, 1} \boldsymbol{c}_{M}^{-1}= \\
{\left[\begin{array}{rr}
0.088 & 0.032 \\
0.032 & -0.059
\end{array}\right] x_{1}+j\left[\begin{array}{r}
0.038-0.072 \\
-0.072-0.026
\end{array}\right] x_{1}}
\end{gathered}
$$

So far, the operations did not introduce any approximation, and the interval remainders are therefore still null and have been omitted. The first approximation occurs in the operation involving the scalar inversion. For the sake of compactness, a first-order representation and the raw estimation of polynomial bounds is used in the following. The aforementioned operation is calculated with the rules of functional expansion outlined in Section IV-C, leading to

$$
\begin{array}{r}
\frac{1}{1+\operatorname{tr}\left(\boldsymbol{c}_{M}^{-1} \overline{\boldsymbol{T}}_{M, 1}\right)}=\left(1-0.096 x_{1}+[0.807,0.960] \times 10^{-2}\right) \\
+j\left(-0.020 x_{1}+[0.336,0.447] \times 10^{-2}\right)
\end{array}
$$

By combining the above results, the first step of the SM algorithm eventually yields the result given in (A.8) at the top of the next page. The application of the second (and in this case last) step of the SM formula involves the calculations in (A.9)-(A.12) and ultimately yields the inverse of $\boldsymbol{M}(\mathrm{x})$ given by (A.13). The element $\left[\boldsymbol{M}^{-1}\right]_{21}$ of this matrix is the TM of the transfer function $T_{H}$ at $\omega=0.61 \mathrm{rad} / \mathrm{s}$. The WC bounds, computed with the raw approach, are $[-0.055,0.385]+$ $[-0.151,0.151]=[-0.206,0.536]$ for the real part, and $[-0.989,-0.581]+[-0.087,0.087]=[-1.076,-0.494]$ for the imaginary part. 


$$
\begin{aligned}
& \left(\boldsymbol{c}_{M}+\overline{\boldsymbol{T}}_{M, 1}\right)^{-1}=\left(\left[\begin{array}{rr}
0.958-0.088 x_{1} & 0.165-0.032 x_{1} \\
0.165-0.032 x_{1} & -0.644+0.059 x_{1}
\end{array}\right]+\left[\begin{array}{ll} 
\pm[0.872] & \pm[0.512] \\
\pm[0.512] & \pm[0.586]
\end{array}\right] \times 10^{-2}\right) \\
& +j\left(\left[\begin{array}{rr}
0.201-0.038 x_{1} & -0.785+0.072 x_{1} \\
-0.785+0.072 x_{1} & 0.685+0.026 x_{1}
\end{array}\right]+\left[\begin{array}{ll} 
\pm[0.624] & \pm[0.715] \\
\pm[0.715] & \pm[0.420]
\end{array}\right] \times 10^{-2}\right) \\
& \left(\boldsymbol{c}_{M}+\overline{\boldsymbol{T}}_{M, 1}\right)^{-1} \overline{\boldsymbol{T}}_{M, 2}=\left(\left[\begin{array}{rr}
0 & -0.239 x_{2} \\
0 & 0.209 x_{2}
\end{array}\right]+\left[\begin{array}{l}
{[0,0] \pm[0.241]} \\
{[0,0] \pm[0.092]}
\end{array}\right] \times 10^{-1}\right)+j\left(\left[\begin{array}{lr}
0 & -0.050 x_{2} \\
0 & 0.196 x_{2}
\end{array}\right]+\left[\begin{array}{l}
{[0,0] \pm[0.112]} \\
{[0,0] \pm[0.198]}
\end{array}\right] \times 10^{-1}\right) \\
& \operatorname{tr}\left(\left(\boldsymbol{c}_{M}+\overline{\boldsymbol{T}}_{M, 1}\right)^{-1} \overline{\boldsymbol{T}}_{M, 2}\right)=\left(0.209 x_{2} \pm[0.092] \times 10^{-1}\right)+j\left(0.196 x_{2} \pm[0.198] \times 10^{-1}\right)
\end{aligned}
$$

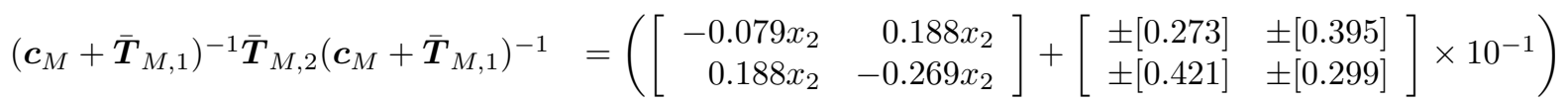

$$
\begin{aligned}
& +j\left(\left[\begin{array}{rr}
0.180 x_{2} & -0.132 x_{2} \\
-0.132 x_{2} & 0.017 x_{2}
\end{array}\right]+\left[\begin{array}{ll} 
\pm[0.407] & \pm[0.356] \\
\pm[0.232] & \pm[0.395]
\end{array}\right] \times 10^{-1}\right) \\
& \frac{1}{1+\operatorname{tr}\left(\left(\boldsymbol{c}_{M}+\overline{\boldsymbol{T}}_{M, 1}\right)^{-1} \overline{\boldsymbol{T}}_{M, 2}\right)}=\left(1-0.209 x_{2}+[-0.021,0.005]\right)+j\left(0.196 x_{2}+[0.055,0.132]\right) \\
& \boldsymbol{M}^{-1}=\left(\left[\begin{array}{rr}
0.958-0.088 x_{1}+0.079 x_{2} & 0.165-0.032 x_{1}-0.188 x_{2} \\
0.165-0.032 x_{1}-0.188 x_{2} & -0.644+0.059 x_{1}+0.269 x_{2}
\end{array}\right]+\left[\begin{array}{ll} 
\pm[0.133] & \pm[0.152] \\
\pm[0.151] & \pm[0.123]
\end{array}\right]\right) \\
& +j\left(\left[\begin{array}{rr}
0.201-0.038 x_{1}-0.180 x_{2} & -0.785+0.072 x_{1}+0.132 x_{2} \\
-0.785+0.072 x_{1}+0.132 x_{2} & 0.685+0.026 x_{1}-0.017 x_{2}
\end{array}\right]+\left[\begin{array}{ll} 
\pm[0.101] & \pm[0.101] \\
\pm[0.087] & \pm[0.148]
\end{array}\right]\right)
\end{aligned}
$$

\section{REFERENCES}

[1] S. R. Nassif, A. J. Strojwas, and S. W. Director, "A methodology for worst-case analysis of integrated circuits," IEEE Trans. Comput.-Aided Des. Integr. Circuits Syst., vol. 5, no. 1, pp. 104-113, Jan. 1986.

[2] W. Tian, X.-T. Ling, and R.-W. Liu, "Novel methods for circuit worstcase tolerance analysis," IEEE Trans. Circuits Syst. I, Fundam. Theory Appl., vol. 43, no. 4, pp. 272-278, Apr. 1996.

[3] M. Tian and C. J. R. Shi, "Worst case tolerance analysis of linear analog circuits using sensitivity bands," IEEE Trans. Circuits Syst. I, Fundam. Theory Appl., vol. 47, no. 8, pp. 1138-1145, Aug. 2000.

[4] L. Kolev, "Worst-case tolerance analysis of linear DC and AC electric circuits," IEEE Trans. Circuits Syst. I, Fundam. Theory Appl., vol. 49, no. 12, pp. 1693-1701, Dec. 2002.

[5] M. Sengupta, S. Saxena, L. Daldoss, G. Kramer, S. Minehane, and J. Cheng, "Application-specfic worst case corners using response surface and statistical models," IEEE Trans. Comput.-Aided Des. Integr. Circuits Syst., vol. 24, no. 9, pp. 1372-1380, Sep. 2005.

[6] R. Spence and R. S. Soin, Tolerance Design of Electronic Circuits. London: Imperial College Press, 1997.

[7] H. Kettani and B. Barmish, "A new Monte Carlo circuit simulation paradigm with specific results for resistive networks," IEEE Trans. Circuits Syst. I, Reg. Papers, vol. 53, no. 6, pp. 1289-1299, Jun. 2006.

[8] L. Scheffer, L. Lavagno, and G. Martin, EDA for IC Implementation, Circuit Design, and Process Technology. Boca Raton, FL: CRC Press, Taylor \& Francis Group, 2006.

[9] P. Triverio, S. Grivet-Talocia, and M. S. Nakhla, "A parameterized macromodeling strategy with uniform stability test," IEEE Trans. Adv. Packag., vol. 32, no. 1, pp. 205-215, Feb. 2009.

[10] D. Deschrijver and T. Dhaene, "Fully parameterized macromodeling of S-parameter data by interpolation of numerator \& denominator," IEEE Microw. Wireless Compon. Lett., vol. 22, no. 6, pp. 309-311, Jun. 2012.

[11] E. R. Samuel, L. Knockaert, F. Ferranti, and D. Dhaene, "Guaranteed passive parameterized macromodeling by using Sylvester state-space realizations," IEEE Trans. Microw. Theory Techn., vol. 61, no. 4, pp. 1444-1454, Apr. 2013.

[12] S. B. Olivadese, G. Signorini, S. Grivet-Talocia, and P. Brenner, "Parameterized and dc-compliant small-signal macromodels of RF circuit blocks," IEEE Trans. Compon. Packag. Manuf. Techol., vol. 5, no. 4, pp. 508-522, Apr. 2015.

[13] D. Vante Ginste, D. De Zutter, D. Deschrijver, T. Dhaene, and F. Canavero, "Macromodeling based variability analysis of an inverted embedded microstrip line," in Proc. IEEE 20th Conf. Elect. Performance Electron. Packaging Syst., San Jose, CA, Oct. 2011, pp. 153-156.
[14] D. Vande Ginste, D. De Zutter, D. Deschrijver, T. Dhaene, P. Manfredi, and F. G. Canavero, "Stochastic modeling-based variability analysis of on-chip interconnects," IEEE Trans. Compon. Packag. Manuf. Techol., vol. 2, no. 7, pp. 1182-1192, Jul. 2012.

[15] Z. Zhang, T. A. El-Moselhy, I. M. Elfadel, and L. Daniel, "Stochastic testing method for transistor-level uncertainty quantification based on generalized polynomial chaos," IEEE Trans. Comput.-Aided Des. Integr. Circuits Syst., vol. 32, no. 10, pp. 1533-1545, Oct. 2013.

[16] M. R. Rufuie, E. Gad, M. Nakhla, and R. Achar, "Generalized Hermite polynomial chaos for variability analysis of macromodels embedded in nonlinear circuits," IEEE Trans. Compon. Packag. Manuf. Techol., vol. 4, no. 4, pp. 673-684, Apr. 2014.

[17] D. Spina, D. De Jonghe, D. Deschrijver, G. Gielen, L. Knockaert, and T. Dhaene, "Stochastic macromodeling of nonlinear systems via polynomial chaos expansion and transfer function trajectories," IEEE Trans. Microw. Theory Techn., vol. 62, no. 7, pp. 1454-1460, Jul. 2014.

[18] P. Manfredi, D. Vande Ginste, D. De Zutter, and F. G. Canavero, "Stochastic modeling of nonlinear circuits via SPICE-compatible spectral equivalents," IEEE Trans. Circuits Syst. I, Reg. Papers, vol. 61, no. 7, pp. 2057-2065, Jul. 2014.

[19] T.-A. Pham, E. Gad, M. S. Nakhla, and R. Achar, "Decoupled polynomial chaos and its applications to statistical analysis of high-speed interconnects," IEEE Trans. Compon. Packag. Manuf. Techol., vol. 4, no. 10, pp. 1634-1647, Oct. 2014.

[20] P. Manfredi and F. Canavero, "Efficient statistical simulation of microwave devices via stochastic testing-based circuit equivalents of nonlinear components," IEEE Trans. Microw. Theory Techn., vol. 63, no. 5, pp. 1502-1511, May 2015.

[21] R. E. Moore, Interval Analysis. Englewood Cliffs, NJ: Prentice-Hall, 1966.

[22] L. H. de Figueiredo and J. Stolfi, "Affine arithmetic: concepts and applications," Numerical Algorithms, vol. 37, no. 1-4, pp. 147-158, Dec. 2004.

[23] T. Ding, R. Trinchero, P. Manfredi, I. S. Stievano, and F. G. Canavero, "How affine arithmetic helps beat uncertainties in electrical systems," IEEE Circuits Syst. Mag., vol. 15, no. 4, pp. 70-79, Nov. 2015.

[24] S. Skelboe, "True worst-case analysis of linear electrical circuits by interval arithmetic," IEEE Trans. Circuits Syst., vol. 26, no. 10, pp. 874879, Oct. 1979.

[25] L. V. Kolev, Interval Methods for Circuits Analysis. Singapore: World Scientific, 1993.

[26] N. Femia and G. Spagnuolo, "Genetic optimization of interval arithmetic based worst case circuit tolerance analysis," IEEE Trans. Circuits Syst. I, Fundam. Theory Appl., vol. 46, no. 12, pp. 1441-1456, Dec. 1999.

[27] N. Femia and G. Spagnuolo, "True worst-case circuit tolerance analysis 
using genetic algorithms and affine arithmetic," IEEE Trans. Circuits Syst. I, Fundam. Theory Appl., vol. 47, no. 9, pp. 1285-1296, Sep. 2000.

[28] J. D. Ma and R. A. Rutenbar, "Fast interval-valued statistical modeling of interconnect and effective capacitance," IEEE Trans. Comput.-Aided Des. Integr. Circuits Syst., vol. 25, no. 4, pp. 710-724, Apr. 2006.

[29] J. D. Ma and R. A. Rutenbar, "Interval-valued reduced-order statistical interconnect modeling," IEEE Trans. Comput.-Aided Des. Integr. Circuits Syst., vol. 26, no. 9, pp. 1602-1613, Sep. 2007.

[30] K. Makino and M. Berz, "Remainder differential algebras and their applications," in Computational Differentiation: Techniques, Applications, and Tools, SIAM, 1996.

[31] M. Berz and G. Hoffstätter, "Computation and application of Taylor polynomials with interval remainder bounds," Rel. Computing, vol. 4, no. 1, pp. 83-97, 1998

[32] K. Makino and M. Berz, "Taylor models and other validated functional inclusion methods," Int. J. Pure Appl. Math., vol. 4, no. 4, pp. 379-456, 2003.

[33] M. Berz and K. Makino, "Verified integration of ODEs and flows using differential algebraic methods on high-order Taylor models," Rel. Computing, vol. 4, no. 4, pp. 361-369, 1998.

[34] Y. Lin and M. A. Stadtherr, "On Taylor model based integration of ODEs," SIAM J. Numerical Anal., vol. 45, no. 1, pp. 236-262, Jan. 2007.

[35] Y. Lin and M. A. Stadtherr, "Validated solutions of initial value problems for parametric ODEs," Appl. Numerical Math., vol. 57, no. 10, pp. 11451162, Oct. 2007.

[36] C.-W. Ho, A. Ruehli, and P. Brennan, "The modified nodal approach to network analysis," IEEE Trans. Circuits Syst., vol. 22, no. 6, pp. 504509, Jun. 1975.

[37] J. Stolfi and L. H. de Figueiredo, "Self-validated reduced numerical methods and applications," in Monograph Brazilian Mathematics Colloquium, IMPA, Rio De Janeiro, Brazil, 1997.

[38] M. A. Abramowitz and I. A. Stegun, Handbook of mathematical functions. New York, NY, USA: Dover Publications, 1970.

[39] W. H. Press, S. A. Teukolsky, W. T. Vetterling, and B. P. Flannery, Numerical Recipes: The Art of Scientific Computing. 3rd edn., New York: Cambridge University Press, 2007.

[40] A. P. Smith, "Fast construction of constant bound functions for sparse polynomials," J. Glob. Optim., vol. 43, pp. 445-458, 2009.

[41] J. Garloff, "Convergent bounds for the range of multivariate polynomials," Proceedings of the International Symp. on interval mathematics on, pp. 37-56, 1985.

[42] J. Garloff, "The Bernstein algorithm", Interval Computations 4, pp. 154 $168,1993$.

[43] G. G. Lorentz, Bernstein Polynomials. New York: Chelsea Publishing Company, 1986

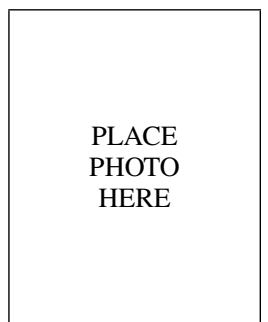

Paolo Manfredi (S'10-M'14) was born in 1985 . He received the M.Sc. degree in electronic engineering from the Politecnico di Torino, Turin, Italy, in 2009, and the Ph.D. degree in information and communication technology from the Scuola Interpolitecnica di Dottorato, Politecnico di Torino, in 2013.

$\mathrm{He}$ is currently a Postdoctoral Research Fellow with the Found for Scientific Research - Flanders (FWO), Electromagnetics Group, Department of Information Technology, Ghent University, Ghent, Belgium. In June 2011 and in February and March 2012, he was a Visiting Scientist with the Center for Advanced Life Cycle Engineering (CALCE), University of Maryland at College Park, MD, USA From April 2012 to July 2012 and in May and June 2013, he was a Visiting Scientist with the Department of Information Technology, Ghent University. From March 2013 to September 2014, he was a Postdoctoral Researcher with the EMC Group, Department of Electronics and Telecommunications, Politecnico di Torino. He authored or co-authored over 50 publications in international journals and conferences proceedings. He regularly serves as a Reviewer for several international journals. His research interests comprise several aspects of circuit and interconnect modeling and simulation, including statistical and worst-case analysis, signal integrity, and electromagnetic compatibility.

Dr. Manfredi was selected for the IBM Best Student Recognition Event in 2009. He was the recipient of the Best Student Paper Award and the Best Oral Paper Award at the 19th and 22nd IEEE Conference on Electrical Performance of Electronic Packaging and Systems (EPEPS) in 2010 and 2013, respectively, and of an Honorable Mention from the IEEE Microwave Theory and Techniques Society (MTT-S) International Microwave Symposium (IMS) in 2011. He was also the recipient of a Young Scientist Award at the XXX General Assembly and Scientific Symposium of the International Union of Radio Science (URSI GASS) in 2011.

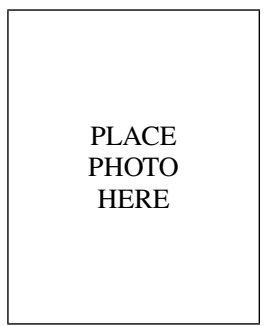

Tongyu Ding (S'15) was born in Heilongjiang, China in 1987. He received the Master degree in communications engineering from Harbin Institute of Technology, China and the Ph.D. degree in electronics and communication engineering from the Politecnico di Torino, Italy, in 2011 and in 2015, respectively. His research interests focus on numerical methods (FDTD) applied to lightning and radiated fields and on the statistical modeling and simulation of electrical and electronic equipment.

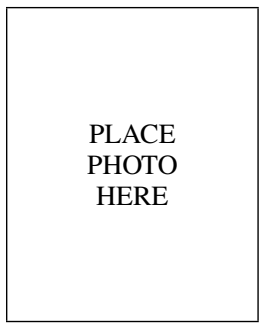

Riccardo Trinchero (S'12-M'16) was born in Casale Monferrato, Italy, in 1987. He received the Master degree and the Ph.D. degree in electronics and communication engineering from Politecnico di Torino, Torino, Italy, in 2011 and in 2015, respectively. He is currently a Postdoctoral Researcher with the Instituto Nazionale di Fisica Nucleare (INFN), sezione di Torino. His research interests include the analysis of linear time-varying systems, modeling and simulation of switching converters, EMC design and statistical simulation of circuits and systems.

\begin{tabular}{|c|} 
\\
\\
PLACE \\
PHOTO \\
HERE \\
\end{tabular}

Igor S. Stievano (M'98-SM'08) received the Master degree in electronic engineering and the Ph.D. degree in electronics and communication engineering from the Politecnico di Torino, Torino, Italy, in 1996 and in 2001, respectively. Currently he is an Associate Professor of Circuit Theory with the Department of Electronics and Telecommunications, Politecnico di Torino. He is the author or coauthor of more than 100 papers published in international journals and conference proceedings. His research interests are in the field of Electromagnetic Compatibility and Signal Integrity, where he works on the modeling and characterization of linear and nonlinear circuit elements. His recent activities include the behavioral modeling of digital ICs, transmission lines and PLC channels, the modeling and simulation of switching converters and the development of stochastic methods for the statistical simulation of circuits and systems with the inclusion of the effects of device or manufacturing uncertainties. 